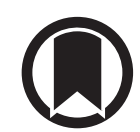

CrossMark

\section{Treatment of multidrug-resistant tuberculosis using therapeutic drug monitoring: first experiences with sub-300 mg linezolid dosages using in-house made capsules}

\author{
To the Editor:
}

Despite all our efforts, the disease burden of tuberculosis (TB) is not falling fast enough to reach the 2030 milestone of the End TB strategy [1]. Multidrug-resistant tuberculosis (MDR-TB) remains a public health crisis, with low treatment success rates [1]. The repurposed drug linezolid has emerged as a core drug in MDR-TB treatment regimens [2, 3], despite its toxicity, e.g. anaemia, peripheral neuropathy and gastrointestinal disorders, optic neuritis, and thrombocytopenia [4, 5]. Currently, linezolid is used off-label, as part of Group A "Medicines to be prioritised" of the World Health Organization (WHO) MDR-TB treatment guideline [2] and in several large trials [6], such as the NIX-TB and END-TB trials.

We use a holistic approach to treat MDR-TB, focusing multidisciplinarily on optimising treatment using pharmacokinetics and pharmacodynamics, assessing and optimising nutritional status, providing physical therapy and social care [7]. Treatment regimens are based on the WHO guidelines, but are individualised based on drug susceptibility testing (DST) combined with TDM if the regimen consists of second-line drugs or if patients are at risk for pharmacokinetics variability [8]. DST is performed at Dutch National Mycobacterial Reference Laboratory (RIVM; Bilthoven, The Netherlands).

Most patients start on a dose of $600 \mathrm{mg}$ linezolid once daily. After 2 weeks, a full pharmacokinetic curve is obtained for all patients that receive linezolid, after which the dosage is lowered to $300 \mathrm{mg}$ once daily. At steady state, the lowered dosage is again checked with TDM. Based on limited available literature, we strive for an area under the time-total fraction concentration curve $\left(\mathrm{AUC}_{0-24 \mathrm{~h}}\right) /$ minimum inhibitory concentration (MIC) ratio >100 [9]. If TDM allows, the dose of linezolid is reduced further to an optimal, lowest dose, with the aim to reduce toxicity of linezolid $[4,5,10]$. If the total $\mathrm{AUC}_{0-24 \mathrm{~h}} / \mathrm{MIC}$ ratio of linezolid is high enough, a dose reduction to 200 or even $150 \mathrm{mg}$ of linezolid once daily is considered. In this study, we describe the first experiences of the treatment of MDR-TB using sub-300 mg dosages.

We retrospectively searched for inpatients for whom linezolid pharmacokinetics data were collected between 2015 and 2018. As routine treatment data were collected retrospectively and anonymously, the Institutional Ethical Review Board waived the requirement to obtain informed consent (METc 2013/492). Patients that received a sub-300 mg dose of linezolid were included. Baseline characteristics co-morbidities (HIV/hepatitis B or C virus/diabetes), MIC for linezolid, treatment regimen and treatment outcome were obtained from the electronic hospital record and the discharge letter from the treating lung physician.

27 patients with linezolid pharmacokinetic data were identified. Of these patients, 18 (66\%) had a final dose $\geqslant 300 \mathrm{mg}$ linezolid once daily. Nine (34\%) patients received a sub-300 $\mathrm{mg}$ dose of linezolid and were included in our study (see table 1). These patients had a median (range) weight of 55 (43-77) $\mathrm{kg}$. At admission, patients had a median (range) leucocyte count $6.6(4.6-10.8) \times 10^{9}$ cells $\cdot \mathrm{L}^{-1}$, thrombocytes 324 $(238-461) \times 10^{9}$ cells $\cdot \mathrm{L}^{-1}$ and haemoglobin $13.2(11.8-16.3) \mathrm{g} \cdot \mathrm{dL}^{-1}$. At admission, one of the patients was

@ERSpublications

Therapeutic drug monitoring allows for sub-300 mg linezolid dosages once daily to successfully achieve PK/PD targets. Let's confirm these retrospective data in a prospective study using dried blood spots. http://bit.ly/2H9fDSc

Cite this article as: Bolhuis MS, van der Werf TS, Kerstjens HAM, et al. Treatment of multidrug-resistant tuberculosis using therapeutic drug monitoring: first experiences with sub-300 $\mathrm{mg}$ linezolid dosages using in-house made capsules. Eur Respir J 2019; 54: 1900580 [https://doi.org/10.1183/13993003.00580-2019]. 
TABLE 1 Baseline characteristics and pharmacokinetic/pharmacodynamic results patients receiving sub-300 $\mathrm{mg}$ dosages of linezolid as a part of their tuberculosis treatment regimens

\begin{tabular}{|c|c|}
\hline Parameter & Value \\
\hline Subjects n & 9 \\
\hline Age years & $27(17-55)$ \\
\hline Male & $4(44)$ \\
\hline Weight kg & $55(43-77)$ \\
\hline Height $\mathrm{m}$ & $1.65(1.55-1.88)$ \\
\hline Body mass index $\mathrm{kg} \cdot \mathrm{m}^{-2}$ & $20.5(16.2-24.1)$ \\
\hline \multicolumn{2}{|l|}{ Continent of origin } \\
\hline Asia & $4(44)$ \\
\hline Europe & $4(44)$ \\
\hline Africa & $1(12)$ \\
\hline HIV, hepatitis B or C positive $n / N$ & 0/9 \\
\hline \multicolumn{2}{|l|}{ Drug susceptibility $n$} \\
\hline Multidrug-resistant & 8 \\
\hline Rifampicin-resistant & 1 \\
\hline \multicolumn{2}{|l|}{ Treatment regimen $n / N$} \\
\hline Linezolid & $9 / 9$ \\
\hline Moxifloxacin & $9 / 9$ \\
\hline Amikacin & $8 / 9$ \\
\hline Ethambutol & $8 / 9$ \\
\hline Clofazimine & $6 / 9$ \\
\hline Pyrazinamide & $5 / 9$ \\
\hline Protionamide & $2 / 9$ \\
\hline (High-dose) isoniazid & $2 / 9$ \\
\hline Cycloserin & $1 / 9$ \\
\hline \multicolumn{2}{|l|}{ Linezolid } \\
\hline \multicolumn{2}{|l|}{ Lowest dose (once daily) } \\
\hline $200 \mathrm{mg}$ & $4(44)$ \\
\hline $150 \mathrm{mg}$ & $5(56)$ \\
\hline Dose per $\mathrm{kg}$ body weight $\mathrm{mg} \cdot \mathrm{kg}^{-1}$ & $3.1(2.0-4.7)$ \\
\hline${ }_{\text {Total }} \mathbf{A U C}_{0-24 \mathrm{~h}} \mathbf{m g} \cdot \mathbf{h} \cdot \mathrm{L}^{-1}$ & $50(35-73)$ \\
\hline $\mathrm{C}_{\min } \mathrm{mg} \cdot \mathrm{L}^{-1}$ & $0.5(0.3-1.2)$ \\
\hline \multicolumn{2}{|l|}{ MIC linezolid $_{\text {S }}$} \\
\hline $0.25 \mathrm{mg} \cdot \mathrm{L}^{-1}$ & 7 (78) \\
\hline $0.5 \mathrm{mg} \cdot \mathrm{L}^{-1}$ & $2(22)$ \\
\hline Total $A \mathrm{CC}_{0-24 \mathrm{~h}} / \mathrm{MIC}$ & $196(98-276)$ \\
\hline
\end{tabular}

Data are presented as median (range) or $\mathrm{n}(\%)$, unless otherwise stated; linezolid concentrations are presented as total fraction concentrations (e.g. total $\mathrm{AUC}$ ); weight, height, and body mass index were collected at admission. MIC: minimum inhibitory concentration; totalAUC: area under the time concentration curve; $\mathrm{C}_{\min }$ : minimum concentration.

32 weeks pregnant. Based on local policy, the individual patient and her preferences, it was decided, in contrast with standard practice, that the start of treatment of MDR-TB could be postponed until the patient gave birth to a healthy child.

Four patients had pulmonary TB, two had extra-pulmonary TB, one had miliary TB, one had pleural TB and one had a combination of pulmonary and extra-pulmonary TB. DST revealed that eight patients had MDR-TB and one had rifampin-resistant TB. The MIC for linezolid of the Mycobacterium tuberculosis isolate was $0.25 \mathrm{mg} \cdot \mathrm{L}^{-1}$ for seven patients and $0.5 \mathrm{mg} \cdot \mathrm{L}^{-1}$ for two patients. Based on the available total $\mathrm{AUC}_{0-24 \mathrm{~h}} / \mathrm{MIC}$ ratios, dosages were lowered to $200 \mathrm{mg}$ once daily in four (15\%) patients and $150 \mathrm{mg}$ in five (19\%) patients.

The sub-300 mg dosages of linezolid are prepared as capsules at the compounding department of our pharmacy. Commercially available linezolid tablets are pulverised and sieved. Lactose-1-water is used to create the correct volume of the homogenised powder mixture, after which transparent empty capsules are filled. All capsules are checked visually and ten capsules are checked for the correct weight and percentage of deviation $(\leqslant 3 \%)$ from the correct weight. The capsules are kept at room temperature for a maximum of 1 year.

With the sub-300 mg dosages of linezolid, the patients had a median (range) total $\mathrm{AUC}_{0-24 \mathrm{~h}}$ of 50 (49-57) $\mathrm{mg} \cdot \mathrm{h} \cdot \mathrm{L}^{-1}$. In three cases, there was no $\mathrm{AUC}_{0-24 \mathrm{~h}}$ available for the lowest dose due to logistical issues. In these cases, the total $\mathrm{AUC}_{0-24 \mathrm{~h}}$ of the lowest dose was calculated using our linezolid pharmacokinetic 
model [11] in MWPharm++ (Mediware, Groningen, the Netherlands). The median (range) totalAUC/MIC ratio was 196 (98-276), with all except one patient attaining the target of 100 that is used in our clinic, based on a systematic review of literature [9]. Since the AUC/MIC ratio of that patient was 98, we did not increase the dose.

As soon as the patients received and tolerated their tailor-made regimen, treatment was continued at home. The included patients finished their treatment as outpatients under supervision of the staff of our centre. At discharge, patients had a median (range) leucocyte count $4.7(3.7-7.3) \times 10^{9}$ cells $\cdot \mathrm{L}^{-1}$, thrombocytes $270(159-327) \times 10^{9}$ cells $\cdot \mathrm{L}^{-1}$ and haemoglobin $13.7(11.1-16.4) \mathrm{g} \cdot \mathrm{dL}^{-1}$. None of the patients had significant adverse events or toxicity from the linezolid dosages they received. Despite the fact that we cannot draw any conclusions about the safety or efficacy of our approach, after discharge, all except one patient (lost to follow-up) finished their treatment regimens successfully.

This study describes the treatment of TB with linezolid dosages lower than $300 \mathrm{mg}$. Linezolid is known for its side effects, with a meta-analysis showing adverse events in $58.9 \%$ of patients with linezolid containing regimens to treat MDR-TB [4]. Higher dosages of linezolid (i.e. $>600 \mathrm{mg}$ ) show more side effects than lower dosages (i.e. between 300 and $600 \mathrm{mg}$ ) [4, 5]. In our study, in nine out of 27 patients, TDM combined with DST allowed for lowering the linezolid dosage to $200 \mathrm{mg}$ or $150 \mathrm{mg}$ once daily, thereby possibly limiting the number of side effects even further.

Obviously, there are limitations to this study, such as the small number of patients, missing data, and the retrospective character of the study. We do not yet have long-term follow-up data; although, so far, no relapses occurred. We only performed pharmacokinetic analysis of blood samples, not of the affected tissue $[12,13]$. Also, the applicability of this study to low-income countries, where MDR-TB is most prevalent, might be limited. For instance, a compounding unit in a pharmacy that is able to produce linezolid capsules might not be readily available. In these cases, one could perhaps consider using quarter tablets of $600 \mathrm{mg}$ linezolid (or $300 \mathrm{mg}$ every 2 days) to administer $150 \mathrm{mg}$ once daily; however, one must then make sure splitting is done in a correct and safe manner. We have not studied the bioequivalence of our capsules. However, since the absorption of linezolid is usually fast and complete, pharmacokinetics of linezolid is non-linear, and we performed TDM, we do not expect this to impact the treatment outcome. Furthermore, TDM is not available everywhere, but dried blood spots (DBS) [14] combined with limited sampling methods [11] might allow for this strategy to be implemented in other settings. There is still an ongoing discussion on the most appropriate pharmacokinetic/pharmacodynamic target for linezolid. In our clinic, we use an AUC/MIC target of $>100$ using the total fraction concentration of linezolid, based on a recent manuscript as a part of a WHO initiative to systematically review the current recommended dose for second-line TB drugs [9]. In future research, besides applicability and implementation of TDM in low-income countries, cost-benefit should also be studied. By administering lower dosages of linezolid in patients if the $\mathrm{AUC}_{0-24 \mathrm{~h}}$ and MIC allows, drug costs per patient of this rather expensive drug is reduced. Finally, lower dosing might reduce dose-related toxicity allowing treatment for a prolonged period of time [15].

In conclusion, sub-300 mg doses of linezolid can achieve putative pharmacokinetic/pharmacodynamic targets in certain patients. A large prospective study with DBS might provide us with valuable information on the possibilities to implement this strategy in other settings.

Mathieu S. Bolhuis ${ }^{1}$, Tjip S. van der Werf ${ }^{2,3}$, Huib A.M. Kerstjens $\oplus^{2,4}$, Wiel C.M. de Lange ${ }^{2,4}$, Jan-Willem C. Alffenaar ${ }^{1,5}$ and Onno W. Akkerman ${ }^{2,4}$

${ }^{1}$ Dept of Clinical Pharmacy and Pharmacology, University of Groningen, University Medical Center Groningen, Groningen, The Netherlands. ${ }^{2}$ Dept of Pulmonary Diseases and Tuberculosis, University of Groningen, University Medical Center Groningen, Groningen, The Netherlands. ${ }^{3}$ Dept of Internal Medicine, University of Groningen, University Medical Center Groningen, Groningen, The Netherlands. ${ }^{4}$ Tuberculosis Center Beatrixoord, University of Groningen, University Medical Center Groningen, Haren, The Netherlands. ${ }^{5}$ Sydney School of Pharmacy, Faculty of Medicine and Health, University of Sydney, Sydney, Australia.

Correspondence: Mathieu S. Bolhuis, Dept of Clinical Pharmacy and Pharmacology, University Medical Center Groningen, University of Groningen, Hanzeplein 1, 9713 GZ Groningen, The Netherlands. E-mail: m.s.bolhuis@umcg.nl

Received: 22 Mar 2019 | Accepted after revision: 12 Aug 2019

Conflict of interest: None declared.

\section{References}

1 World Health Organization (WHO). Global Tuberculosis Report 2018. Geneva, WHO, 2018.

2 World Health Organization (WHO). Rapid Communication: Key changes to treatment of multidrug- and rifampicin-resistant tuberculosis (MDR/RR-TB). Geneva, WHO, 2018.

3 Caminero JA, Piubello A, Scardigli A, et al. Proposal for a standardised treatment regimen to manage pre- and extensively drug-resistant tuberculosis cases. Eur Respir J 2017; 50: 1700648. 
4 Sotgiu G, Centis R, D’Ambrosio L, et al. Efficacy, safety and tolerability of linezolid containing regimens in treating MDR-TB and XDR-TB: systematic review and meta-analysis. Eur Respir J 2012; 40: 1430-1442.

5 De Lorenzo S, Alffenaar JW, Sotgiu G, et al. Efficacy and safety of meropenem/clavunate added to linezolid containing regimens in the treatment of M/XDR-TB. Eur Respir J 2013; 41: 1386-1392.

6 Borisov SE, Dheda K, Enwerem M, et al. Effectiveness and safety of bedaquiline-containing regimens in the treatment of MDR- and XDR-TB: a multicentre study. Eur Respir J 2017; 49: 1700387.

7 Akkerman OW, Grasmeijer F, de Lange WCM, et al. Cross border, highly individualised treatment of a patient with challenging extensively drug-resistant tuberculosis. Eur Respir J 2018; 51: 1702490.

8 Van Kampenhout E, Bolhuis MS, Alffenaar JC, et al. Pharmacokinetics of moxifloxacin and linezolid during and after pregnancy in a patient with multidrug-resistant tuberculosis. Eur Respir J 2017; 49: 1601724.

9 Bolhuis MS, Akkerman OW, Sturkenboom MGG, et al. Linezolid-based regimens for multidrug-resistant tuberculosis (TB): a systematic review to establish or revise the current recommended dose for TB treatment. Clin Infect Dis 2018; 67: S327-S335.

10 Yew WW, Chan DP, Leung CC, et al. Can toxicities induced by antituberculosis drugs be better managed in diabetic patients? Eur Respir J 2017; 50: 1700409.

11 Kamp J, Bolhuis MS, Tiberi S, et al. Simple strategy to assess linezolid exposure in patients with multi-drug-resistant and extensively-drug-resistant tuberculosis. Int J Antimicrob Agents 2017; 49: 688-694.

12 Akkerman OW, van Altena R, Klinkenberg T, et al. Drug concentration in lung tissue in multidrug-resistant tuberculosis. Eur Respir J 2013; 42: 1750-1752.

13 Kempker RR, Heinrichs MT, Nikolaishvili K, et al. A comparison of linezolid lung tissue concentrations among patients with drug-resistant tuberculosis. Eur Respir J 2018; 51: 1702166.

$14 \mathrm{Vu}$ DH, Bolhuis MS, Koster RA, et al. Dried blood spot analysis for therapeutic drug monitoring of linezolid in patients with multidrug-resistant tuberculosis. Antimicrob Agents Chemother 2012; 56: 5758-5763.

15 van Altena R, Akkerman OW, Alffenaar JC, et al. Shorter treatment for multidrug-resistant tuberculosis: the good, the bad and the ugly. Eur Respir J 2016; 48: 1800-1802. 